\title{
Nutricionalmente hablando, ¿el queso Oaxaca Light es diferente al queso convencional?
}

\section{Nutritionally speaking, is Oaxaca Light cheese different from a conventional cheese?}

\author{
Lizeth M. Isidro-Bernal ${ }^{a}$, Kimberly Moreno-Gutiérrez ${ }^{b}$, Juan E. Téllez-Delgadillo ${ }^{c}$, Eduardo \\ Hernández-Díaz ${ }^{d}$, Laura Pontaza-Ramos ${ }^{e}$
}

\begin{abstract}
:
Oaxaca cheese is a soft and plastic curd cheese, crumbly, with a firm body, creamy white color, soft flavor and slightly acidic. The main characteristic is its elastic consistency, since the curd can be molded into a round and braided shape. Due to the high fat content the dairy industry has innovated with the production of Oaxaca Light cheeses as an alternative in low calorie products. On the other hand, the high content of sodium that characterizes these products allows to maintain an adequate texture, besides contributing in its conservation. The purpose of this work was to establish a nutritional comparison based on the information presented in the labeling of Oaxaca and Oaxaca Light cheeses that are sold in the city of Pachuca Hidalgo. 11 brands of Oaxaca cheese and 3 brands of Oaxaca Light cheese were evaluated, gathering the nutritional information and additives to make a comparison on their nutritional composition. Both in the conventional Oaxaca and Light cheeses covered the protein content (>20\%), however, the fat content was adequate only in the conventional cheeses (it should not be less than 18\%) according to the Regulation of Sanitary Control of Products and Services, which regulates these products, however, those products that presented a low fat content were used gums as an additive. The Light cheeses, compared to the conventional cheeses, presented a low lipid content, and therefore caloric, reason why they can be considered as a beneficial option within the feeding.
\end{abstract}

Keywords:

Oaxaca Light cheese, Oaxaca cheese, nutritional information, additives.

\section{Resumen:}

El queso Oaxaca es un queso de cuajada suave y plástica, desmenuzable, de cuerpo firme, color blanco cremoso, sabor suave y ligeramente ácido. La característica principal es su consistencia elástica, ya que la cuajada se puede moldear hasta darle una forma redonda y trenzada. Debido al alto contenido de grasas la industria láctea ha innovado con la producción de quesos Oaxaca Light como una alternativa en productos de bajo contenido calórico. Por otro lado, el alto contenido de sodio que caracteriza a estos productos permite mantener una textura adecuada, además de contribuir en su conservación. El propósito del presente trabajo fue establecer una comparación nutrimental con base en la información presentada en el etiquetado de quesos Oaxaca y quesos Oaxaca Light que se expenden en la ciudad de Pachuca Hidalgo. Se evaluaron 11 marcas de queso Oaxaca y 3 marcas de queso Oaxaca Light, recopilando la información nutrimental y aditivos para realizar una comparación sobre su composición nutrimental. Tanto en los quesos Oaxaca convencional y Light cubrieron el contenido proteico (>20\%), sin embargo, el contenido de grasa fue lo adecuado solo en los quesos convencionales (no debe ser menor al 18\%) de acuerdo con el Reglamento de Control Sanitario de Productos y Servicios, que regulan estos productos, sin embargo, aquellos productos que presentaron un bajo contenido de grasa se utilizaron gomas como

\footnotetext{
Autor de correspondencia, Universidad Autónoma del Estado de Hidalgo, https://orcid.org/0000-0002-3248-6653, Email: lizeth_berna105@hotmail.com

b Universidad Autónoma del Estado de Hidalgo, https://orcid.org/0000-0001-6114-038X, Email: kimberlymgtz@hotmail.com c Universidad Autónoma del Estado de Hidalgo, https://orcid.org/0000-0002-5999-4658, Email: estebantelleznutricion@gmail.com d Universidad Autónoma del Estado de Hidalgo, https://orcid.org/0000-0002-1612-4409, Email: eduardo.hernandez.diaz98@gmail.com e Universidad Autónoma del Estado de Hidalgo, https://orcid.org/0000-0002-5196-661X, Email: lauramos412@gmail.com
} 
aditivo. Los quesos Light comparado con los quesos convencionales, presentaron un bajo contenido lipídico, y por ende calórico, por lo que pueden ser considerados como una opción beneficiosa dentro de la alimentación.

\section{Palabras Clave:}

Queso Oaxaca Light, queso Oaxaca, información nutrimental, aditivos.

\section{Introducción}

De acuerdo con la NOM-243-SSA-2010', se refiere al queso como producto elaborado con la cuajada de leche estandarizada y pasteurizada de vaca o de otras especies animales, obtenida de la coagulación de la caseína con cuajo, entre otros, está compuesto por agua, grasa, proteínas, minerales y sales, los cuales están asociados con las características fisicoquímicas de la leche ${ }^{2}$.

El queso Oaxaca, también conocido como quesillo o queso de hebra es definido como un producto de cuajada suave y plástica, desmenuzable, de cuerpo firme, color blanco cremoso y sabor suave, ligeramente ácido. La característica principal es su consistencia elástica, ya que la cuajada se puede moldear hasta darle una forma redonda y trenzada ${ }^{3}$.

Por estas características es considerado uno de los alimentos más consumidos por niños y adultos, sin embargo, tiene un gran contenido en grasa y sal. Por lo que la industria presenta algunas otras alternativas como los quesos Oaxaca Light, productos con bajo contenido de grasa y por lo tanto, de calorías. La utilización de leche descremada ocasiona modificación en la textura de los quesos por lo que se obtienen productos más firmes, duros, secos y granulados 4 .

El contenido de sal en los quesos Oaxaca es alto, al ser un elemento necesario, ya que afirma la textura y tiene efectos sobre su conservación, como resultado de la eliminación de agua necesaria para alcanzar los niveles de humedad requeridos para la presión de la cuajada durante su elaboración y obtener un prensado ideal ${ }^{4}$.

\section{Metodología}

Realizar una comparación nutrimental con base en la tabla nutricional presente en el etiquetado de las diferentes marcas comerciales de los quesos Oaxaca convencionales y Oaxaca Light expendidos en comercios convencionales de la ciudad de Pachuca Hidalgo, permitiendo determinar la opción del producto más favorable para una alimentación saludable.

Se analizaron 11 marcas de queso Oaxaca y 3 marcas del producto Light. Los productos fueron obtenidos en comercios de mayor importancia de la ciudad de Pachuca de Soto.
La información nutrimental (Kcal, cantidad de proteína, lípidos, carbohidratos, sodio) y aditivos fue recopilada en una base de datos y analizada para una comparación con parámetros establecidos por normas mexicanas sobre su composición nutrimental, calculados con base a $100 \mathrm{~g}$ para poder establecer las comparaciones.

En la Tabla 1 se observa la información nutrimental de las diferentes marcas revisadas de queso Oaxaca y la versión Light. Acorde a la información recabada, se muestra que el contenido calórico del queso Oaxaca convencional varía entre 260 a $330 \mathrm{kcal}$, mientras que en el Light va de 213.3 a $260 \mathrm{kcal} / 100 \mathrm{~g}$ de producto, mostrando una disminución de $46.7 \mathrm{kcal}$ respecto al queso Oaxaca. Para lograr un bajo contenido calórico en dichos productos se utiliza leche descremada en su elaboración ${ }^{5}$, lo que influye en su contenido lipídico.

Aunque este producto es uno de los más comercializados en México, no existe una norma específica, por lo que se aplica el Reglamento de Control Sanitario de Productos y Servicios ${ }^{3}$. Como guía para el proceso de producción especifica que, para los quesos frescos de pasta cocida e hilada, la cantidad de proteína debe ser $>20 \%$ y la cantidad de grasa no debe ser menor al $18 \%$. La mayoría de los quesos tienen un aporte proteico entre 21 a $26 \mathrm{~g}$ y cumplen con lo especificado en el Reglamento, a excepción de tres marcas que presentan un menor porcentaje (entre 14 y $16 \mathrm{~g}$ ). Mientras que el contenido de grasas en todos los quesos Oaxaca convencionales (20.1 g a $26.6 \mathrm{~g} / 100 \mathrm{~g}$ ) revisados cumplen con el reglamento mencionado anteriormente de contenido en grasa (mínimo 18\%), a excepción de los productos "La Villita®", "Nochebuena Trenzado Artesanal $₫$ ", "Flor de Alfalfa $® "$ que tienen un contenido mucho menor a lo establecido. Los quesos Light no estarían legislados con dicho reglamento por lo que el contenido de grasa es mucho menor y está permitido (entre 4 a $13 \mathrm{~g}$ de lípidos/100 g de producto).

La actualización de la NOM-051-SCFI/SSA12010 tiene el objetivo de evaluar el etiquetado nutricional de alimentos y bebidas comercializados, de igual manera determinar las características de dicha información la cual debe advertir de forma clara sobre el contenido en exceso de calorías, grasas, azúcares y sodio que representan un riesgo para la salud. Dicha norma establece que si el contenido de sodio es $>350 \mathrm{mg} / 100 \mathrm{~g}$ de producto, el 
etiquetado deberá presentar un sello de advertencia "exceso de sodio" y si el producto tiene un contenido mayor de $275 \mathrm{Kcal} / 100 \mathrm{~g}$ debe tener un sello de advertencia de "exceso de energía".

Dentro de las marcas evaluadas la cantidad de sodio añadido en ambas presentaciones sobrepasa, por ello es necesario la presencia de dicho sello de advertencia, lo mismo que para el contenido de grasa en todos los productos con excepción de las marcas evaluadas, (a excepción de "Alpura®", "Esmeralda®" y "Flor de Alfalfa $®$ " y quesos Light) deben presentar dicha advertencia.

Tabla 1. Información nutrimental del queso Oaxaca y Light (g/100 g).

\begin{tabular}{|c|c|c|c|c|c|}
\hline Producto & 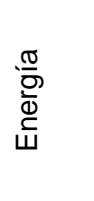 & 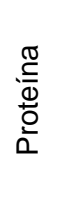 & $\begin{array}{l}\text { 음 } \\
\text { 음 }\end{array}$ & 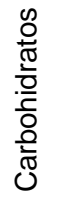 & 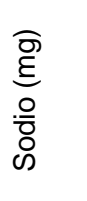 \\
\hline \multicolumn{6}{|c|}{ Quesos Oaxaca } \\
\hline Lala & 283.3 & 24 & 20 & 1.6 & 633.3 \\
\hline Zwan premium & 283.3 & 22 & 21.3 & 1 & 630 \\
\hline Fud & 293.3 & 22.6 & 21.6 & 1.6 & 630 \\
\hline Alpura & 260 & 23 & 18 & 1.6 & 700 \\
\hline Los Volcanes & 273.3 & 29 & 26.6 & 2 & 650 \\
\hline La Villita & 303.3 & 14 & 14.8 & 0.6 & 666.6 \\
\hline Nochebuena & & & & & \\
\hline trenzado artesanal & 293.3 & 22.6 & 13.3 & 2 & 630 \\
\hline Covadonga & 295.6 & 23.6 & 20.1 & 4.9 & 169.3 \\
\hline Esmeralda & 273.3 & 16.6 & 41.4 & 2 & 560 \\
\hline Great Value & 330 & 14 & 26.3 & 2.3 & 873.3 \\
\hline Flor de Alfalfa & 273.1 & 28.1 & 16.1 & 3.9 & 373 \\
\hline \multicolumn{6}{|c|}{ Quesos Oaxaca Light } \\
\hline Lala & 213.3 & 26 & 11 & 2.6 & 633.3 \\
\hline Esmeralda & 231 & 22 & 4.3 & 2 & 560 \\
\hline Great Value & 260 & 21 & 13 & 4 & 660 \\
\hline
\end{tabular}

En la Tabla 2, se observan los aditivos utilizados para la producción de los quesos evaluados. Los quesos Oaxaca convencionales presentaron un mayor contenido de aditivos para mantener el color y la textura característica del producto.

Según la CODEX-STAN 192-19957, los aditivos alimentarios son sustancias añadidas al alimento con fines tecnológicos en su fase de elaboración y/o producción. Uno de los aditivos más comúnmente utilizado en la elaboración del queso Oaxaca en las dos presentaciones mencionadas, es el cuajo, que tiene la propiedad de cuajar la caseína contenida en la leche ${ }^{8}$; mientras mayor sea la cantidad de cuajo agregado, con mayor rapidez coagula la leche, hay incremento de volumen y estabiliza a las propiedades.
Tabla 2. Comparación de aditivos utilizados en las diferentes marcas de queso Oaxaca

\begin{tabular}{|c|c|c|c|c|c|}
\hline Marca & 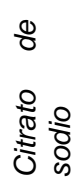 & 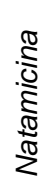 & 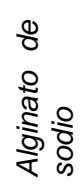 & 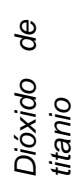 & 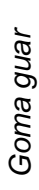 \\
\hline \multicolumn{6}{|c|}{ Quesos Oaxaca } \\
\hline Lala & & $\mathrm{X}$ & \multirow{7}{*}{$X$} & \multirow{8}{*}{$\begin{array}{l}X \\
X\end{array}$} & \multirow{8}{*}{$\begin{array}{l}X \\
X\end{array}$} \\
\hline Zwan premium & & $\mathrm{X}$ & & & \\
\hline & $X$ & & & & \\
\hline Los Volcanes & & $x$ & & & \\
\hline La Villita & & & & & \\
\hline $\begin{array}{l}\text { Nochebuena } \\
\text { trenzado artesanal } \\
\text { Covadonga }\end{array}$ & & $X$ & & & \\
\hline Esmeralda & & & & & \\
\hline $\begin{array}{l}\text { Great Value } \\
\text { Flor de Alfalfa }\end{array}$ & $x$ & $\mathrm{X}$ & & & \\
\hline \multicolumn{6}{|c|}{ Quesos Oaxaca Light } \\
\hline Lala & & $\mathrm{X}$ & & & \\
\hline Esmeralda & & $\mathrm{X}$ & & & \\
\hline Great Value & $\mathrm{X}$ & & & & \\
\hline
\end{tabular}

Conforme a la normativa "Acuerdo por el que se determinan los Aditivos y Coadyuvantes en alimentos, su uso y disposiciones sanitarias" ", mencionados en la tabla 2, algunos de estos son empleados a través las Buenas Prácticas de Fabricación (BPF), en el que se limitará a la dosis mínima necesaria para obtener el efecto deseado, por ejemplo, la Natamicina, utilizado para extender la vida útil del producto, el cual actúa como conservador. El citrato de sodio funciona como emulsificante y el alginato de sodio como incrementador de volumen y ambos son permitidos de acuerdo a la normativa.

Respecto a la goma guar, comúnmente se presenta en este tipo de queso, mostrando adición especialmente en "La Villita®" y "Nochebuena Trenzado Artesanal $\AA$ ". El aditivo está aprobado por la Administración de Medicamentos y Alimentos (FDA, por sus siglas en inglés) como ingrediente opcional en la elaboración de quesos, permitiendo hasta el $3 \%$ del peso total, para dar al producto una mejora en el rendimiento de sólidos de cuajada; su adición permite la elaboración de quesos bajos en grasa sin cambiar la reología y textura original $^{10}$. Por lo que es llamativo que los quesos Light no presenten este aditivo entre sus ingredientes, a pesar de tener un bajo contenido de grasa.

Dentro del etiquetado de ingredientes de cada uno de los productos, se encontró el aditivo llamado dióxido de titanio, el cual es utilizado para dar color al alimento, generalmente color blanco brillante, ya que pueden surgir pérdidas durante el proceso industrial; este solo fue identificado en dos de los productos mostrados en la tabla 2 ("Nochebuena Trenzado Artesanal囚" y 
"Covadonga®"), los cuales, pueden llegar a presentar mayor vivez de color y textura adecuada al queso Oaxaca ${ }^{11}$.

\section{Conclusión}

Los quesos Oaxaca convencional como los Light, muestran diferencias respecto a su composición nutrimental. Los quesos Light sin duda resultan la opción más favorable dentro de la alimentación, dado que principalmente presentan una disminución en su contenido lipídico y por ende calórico, comparado a los quesos convencionales. Sin embargo, su consumo debe ser moderado debido a su elevado contenido en sodio que indican ambos tipos de queso.

\section{Referencias}

[1] NOM-243-SSA1-2010. Productos y servicios. Leche, fórmula láctea, producto lácteo combinado y derivados lácteos. Disposiciones y especificaciones sanitarias. Métodos de prueba. En: http://dof.gob.mx/normasOficiales/4156/salud2a/salud2a.htm

[2] Ramírez, S. \& Vélez, J. Queso Oaxaca: panorama del proceso de elaboración, características fisicoquímicas y estudios recientes de un queso típico mexicano. Temas Select Ing Alim. 2012; 6(1): 1-12.

[3] Reglamento de Control Sanitario de Productos y Servicios. En http://www.salud.gob.mx/unidades/cdi/nom/compi/rlgsmcsaeps.html

[4] Mayta, H. J., Trujillo, A. J. \& Juan, B. Tecnología de quesos en grasa. Rev, Investig. Vet. 2019: 30(4): 1382-1394.

[5] Bello, J.M., Lizeldi, B., González, E., Manzo, A., Nochebuena, X., Quiñones, E.I. \& Vázquez, C. Productos lácteos: la ruta de la metamorfosis. Revista Digital Universitaria. 2004: 5(7): 1-14.

[6] NOM-051-SCFI/SSA1-2010. Especificaciones generales de etiquetado para alimentos y bebidas no alcohólicas pre-envasados. Información comercial sanitaria. En: http://www.dof.gob.mx/normasOficiales/4010/seeco11_C/seeco11_C. htm

[7] CODEX-STAN 192-1995. Norma General para los Aditivos Alimentarios. En: http://www.fao.org/gsfaonline/docs/CXS_192s.pdf

[8] Guzmán, V. Procesamiento de quesos. REDAR. 2012; 7(1): 31-34.

[9] Acuerdo por el que se determinan los Aditivos y Coadyuvantes en alimentos, su uso y disposiciones sanitarias. En: https://www.dof.gob.mx/nota_detalle_popup.php?codigo=5259470\#: $\sim$ :text=Para\%20los\%20aditivos\%20que\%20se,el\%20Ap\%C3\%A9ndi ce $\% 20$ del $\% 20$ Reglamento $\% 20 \mathrm{de}$

[10] Castañeda, A., González, L.A., Granados, M.A. \& Chávez, U.J. Goma Guar: Un aliado en la Industria Alimentaria. Pädi. Boletín Científico de Ciencias Básicas e Ingenierías del ICBI. 2019; 7(14): 107-111.

[11] Sánchez, J. La química del color en los alimentos. Química Viva. 2013; 12(3): $234-246$ 